\title{
Artificial Immune Algorithm-based Airplane Path Planning under Complicated Environment
}

\author{
Lifeng Liu \\ School of architectural engineering, Shandong University of Technology, Zibo 255000, Shandong, \\ China \\ Zongwei Niu \\ School of mechanics engineering, Shandong University of Technology, Zibo 255000, Shandong, China
}

Received: January 27, 2021. Revised: July 6, 2021. Accepted: July 28, 2021. Published: July 30, 2021.

\begin{abstract}
This paper mainly studies the flight route planning in complex and uncertain environments. The improved artificial immune algorithm is used to complete the flight route planning in different complex environments. The experimental results show that the improved artificial immune algorithm has a higher success rate of route planning than the common genetic algorithm in complex environments; therefore, it also shows that this method has better adaptability in complex environment.
\end{abstract}

Keywords-Airplane, AIA-based Path Planning, Algorithm comparison, uncertain environment.

\section{INTRODUCTION}

$I^{T}$ $\mathrm{T}$ is very important to plan a safe and operational path for an aircraft in a rescue mission under an environments including terrain and building threats. Currently, multiple UAVs cooperate with each other to complete the rescue task $[1,2]$. Therefore, the design of multi Aircraft Cooperative Route Planning in complex environment has great application value in civil rescue fields.

In the field of path planning, many methods have been developed in a given environment. Graph search method can be used to generate a planar path in short time under the environment with known threat information [3, 4]. A * algorithm usually has better performance such as accuracy and velocity with the help of heuristic function, but the path planned can be local optimal solution $[5,6]$. Ant colony algorithm is a heuristic global optimization algorithm. However, this method's use is limited due to its relatively slow convergence speed and tendency to be trapped in local minim [7]. Evolutionary algorithms have inherent parallelism and good global search capabilities, but its local search ability is poor, prone to the premature convergence [8]. The potential field theory is a simple, easy and low-level algorithm, while it is prone to stuck in local minima and deadlocking [9]. Artificial neural networks (ANN) have the ability of quickly determine optimal solutions, but it requires a number of parameters and too long learning time to reach the desired destination [10]. The bacterial memetic algorithm has also been proposed to solve the path-planning problem in uncertain environments [11, 12]. Particle Swarm Optimization (PSO) method is also been used in path planning[13], PSO A new hybrid PSO known as the HGC-PSO uses Gaussian and Cauchy mutations to increase the population diversity and avoid the tendency of the PSO to be stuck on local optima values [14]. A hybrid path planning algorithm for the $\mathrm{D}^{*}$ algorithm and Voronoi graph has been developed that incorporates global and local path planning schemes for mobile robots [15]. Genetic algorithm (GA) has good global searching ability when it is used in route planning, but it also has the problem of premature convergence [16].

Based on the basic AIA algorithm, this paper develops an AIA algorithm for aircraft route planning. Combined with the various threat scenarios in the rescue mission, the basic AIA algorithm is improved by introducing the aircraft maneuverability and flightability optimization module to improve the safety of the planned route. Aiming at the situation of multi Aircraft Cooperative mission, this paper designs the dual aircraft route planning AIA algorithm: introduces the voyage comparison module to reduce the voyage difference between the two aircraft, so as to ensure that the two aircraft can reach the destination at the same time and improve the efficiency of mission execution. Compared with the widely used genetic algorithm, experiments are carried out in many complex environments. The experimental results show that the algorithm has better operability and robustness.

\section{AIRCRAFT PATH PLANNING PROBLEM FORMULATION}

The purpose of this paper is to calculate a $3 \mathrm{D}$ flight path using the AIA with the desired characteristics of aircraft in a complicated environment. For the multiple aircraft path planning problem, there are two aspects that must be taken into account: (1) the cooperative problem in multiple aircraft path planning and (2) multiple aircraft cooperating with each other during the flight planning process. For multiple aircraft systems, the main problem is the cooperation of multiple aircraft, the arrival time, direction of attack and the task 
assignments.

Aircraft path planning assumed in this paper has characteristics including:

(1) Stealth. The designed path must minimize threats by choosing radar shadow zones, maximize a safety margin from ground obstacles and constrain the maximum and minimum elevation from the ground.

(2) Physical feasibility and mission performance. This is related to special aircraft performance, such as the maximum climb and descent rates, flight path angles, the length of the flight path, airspeed and minimum turning radius.

(3) Estimated time of arrival (ETA). For multi-aircraft path planning, all aircraft must arrive at the destination at almost at the same time to complete a certain task.

(4) Multiple aircraft cooperation. When an unexpected threat appears, aircrafts should safely reach target region in the shortest time by information exchange.

(5) Real-time implementation. This requires that the design time of the next flight segment be less than the execution time of present flight segment.

\section{ARTIFICIAL IMMUNE ALGORITHM-BASED PATH PLANNING}

\section{A. Artificial Immunity Algorithm (AIA)}

AIA refers to the concept and theoretical calculation model of biological immune system. It bases the immune algorithm to be solved on the biological humoral immune process. The characteristics of biological fluid immunity includes antigen recognition, plasma cells selected by affinity, having memory cells, promoting and inhibiting the production of antibodies, and generating the next generation of antibodies through cross mutation.

The AIA is capable of high level parallel processing, and does not only have learning and memory capabilities but also has related repair, distribution and self-organization abilities. Therefore, AIA provides a new way to yield intelligent control in this particular research field.

Our research utilizes the AIA's advantages and combines the method with aircraft path planning. At the same time, we consider the aircraft's airworthiness, namely, the condition of the aircraft with respect to its aerodynamic, mechanical and legal ability to fly.

\section{B. Main techniques of AIA aircraft path planning}

Because AIA is seldom used in aircraft path planning field, we provide a description of AIA's operation as follows.

\section{1) Spatial discretization}

The planning space is continuously distributed, and the track points in the flight route are discrete. In order to facilitate the selection of the track points, it is necessary to discretize the planning space. At the same time, ground surface model (DTM) also need to be spatialized base on the granularity of airspace to unify calculation of route scale (see Figure 1). The granularity of airspace's discretization has an important impact on the calculation efficiency and memory capacity, which just like the higher the resolution of the image, the larger the storage space. In this paper, a concept is explained for airspace's grid discretization in a rough path set theoretic planning. Formula (1) and formula (2) show the 3D discrete dot set formed.

$$
\begin{gathered}
P_{s p}(i, j)=P_{s p}\left(x_{s p}(i, j), y_{s p}(i, j), h_{s p}(i, j)\right) \\
i=1,2, \ldots N_{s p}, j=1,2, \ldots M_{s p}, h_{\text {limit } \_ \text {high }} \geq h_{s p}(i, j)>h_{D T M} \\
M_{s p}=\left[W_{s p} / D_{w}\right], N_{s p}=\left\lceil L_{s p} / D_{l}\right\rceil
\end{gathered}
$$

Here, $P_{s p}(i, j)$ and $\left(x_{s p}(i, j), \mathrm{y}_{s p}(i, j), h_{s p}(i, j)\right)$ indicate the point of $i$ th row and $j$ th column, corrdination on the airspace respectively, with unit being per kilometer, $i \in\left(1,2, \ldots, M_{s p}\right), j$ $\in\left(1,2, \ldots, N_{s p}\right), h_{s p} . W_{s p}, L_{s p}$ repress the width and Length of airspace respectively; symbol ' $\rceil$ ' indicates rounding up. $D_{w}$ and $D_{l}$ are the discreted distance of $x$-axis, $y$-axis on level airspace respectively. $h_{\text {limit }}$ expresses airspace's the limit height of planning, which include high limit (i.e. $h_{\text {limit_high }}$ ) and low limint (i.e. $\left.h_{\text {limit_low }}\right)$, that is, $h_{\text {limit }} \in\left(h_{\text {limit_high }}, h_{\text {limit_low }}\right)$. $h_{D T M}$ means the height of DTM in formula (3).

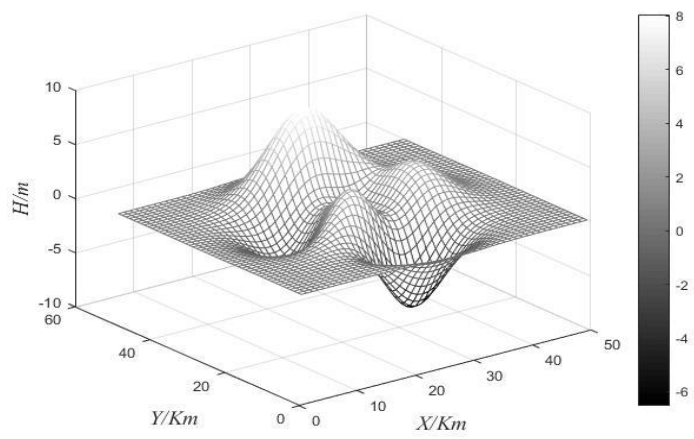

(a)

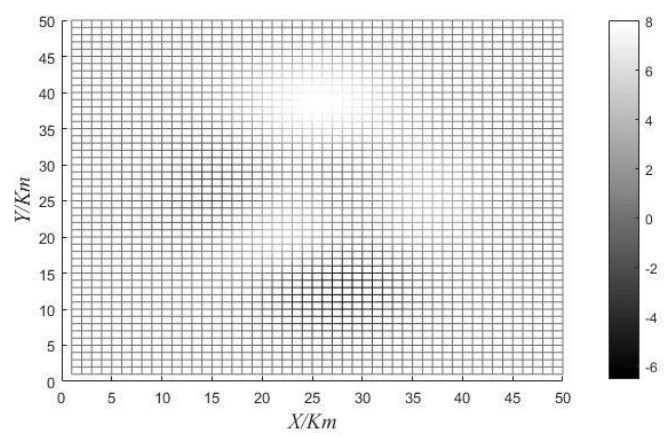

(b) 


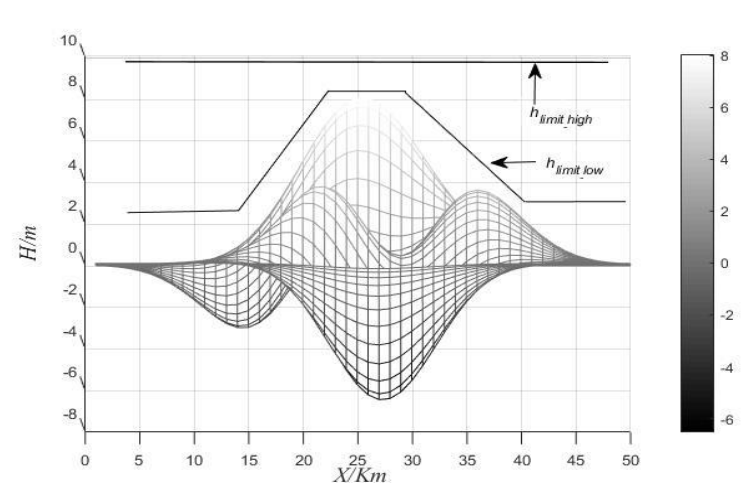

(c)

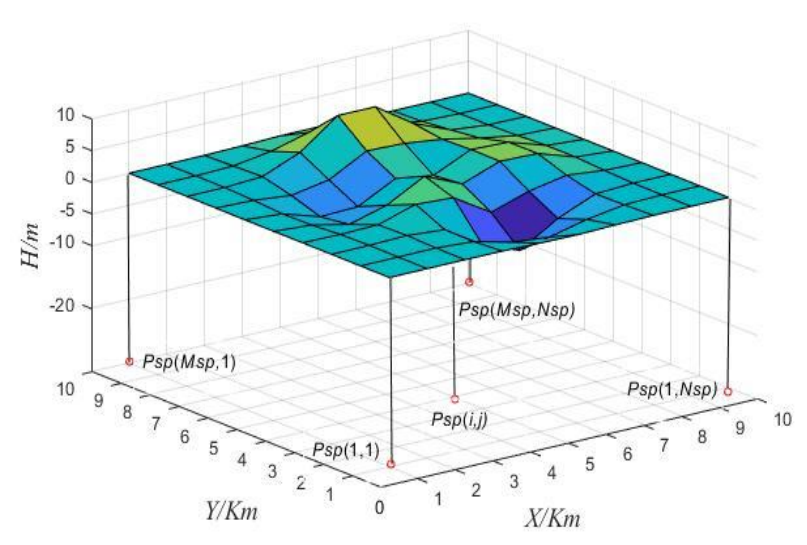

(d)

Fig. 1. Discrete DTM (a), DTM projection on $x-y$ plane (b) and $y-h$ plane(c) and a real DTM and its projection on $x-y$ plane (d)

$$
h_{D T M}=\left[\begin{array}{cccccc}
h_{D T M}(1,1) & h_{D T M}(1,2) & \ldots & h_{D T M}(1, j) & \ldots & h_{D T M}\left(1, M_{s p}\right) \\
\left.h_{D T M}(2,1)\right) & h_{D T M}(2,2) & \ldots & h_{D T M}(2, j) & \ldots & h_{D T M}\left(2, M_{s p}\right) \\
\vdots & \vdots & \ldots & \vdots & \ldots & \vdots \\
h_{D T M}(i, 1) & h_{D T M}(i, 2) & \ldots & h_{D T M}(i, j) & \ldots & h_{D T M}\left(i, M_{s p}\right) \\
\vdots & \vdots & \ldots & \vdots & \ldots & \vdots \\
h_{D T M}\left(M_{s p}, 1\right) & h_{D T M}\left(M_{s p}, 2\right) & \ldots & h_{D T M}\left(M_{s p}, j\right) & \ldots & h_{D T M}\left(M_{s p}, N_{s p}\right)
\end{array}\right]
$$

2) Route expression

A flight route $(F)$ is composed of a track points $\left(P_{f}\right)$ set in airspace. $P_{f}(k, l)$ is the $l^{\text {th }}$ point of $k^{\text {th }}$ flight path, which corrdination is expressed as $\left(x_{f}, y_{f}, h_{f}\right) \quad(k, l)$, which is simplified form of $x_{f}(k, l), y_{f}(k, l), h_{f}(k, l), l \in\left(1,2, \ldots, N_{f}\right), k \in$ $\left(1,2, \ldots, N_{p}\right)$ in formula (4)and formula (5). $N_{p}$ and $N_{f}$ indicate the number of flight path, flight point respectively. $h_{\text {suitability }}$ means the value more suitable for plane safe flight, which is in the range of $h_{\text {suitability_low }}$ and $h_{\text {suitability_high }}$. $h_{\text {plane_adjust }}$ expresses the safety margin value adjusted for the sake of safety (see figure 2).

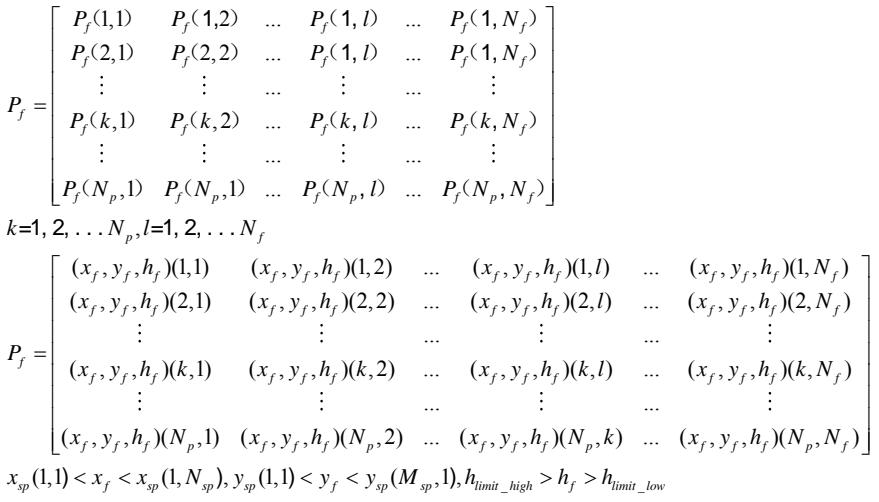

$h_{f} \subset h_{\text {suitability }}=\left\{h_{\text {suitability_low }}, h_{\text {suitability_high }}\right\}$

$h_{\text {suitability_low }}=h_{D T M}+h_{\text {min_flight_altitude }}+h_{\text {plane_adjust }}, h_{\text {suitability_high }}$

or, $h_{\text {suitability_low }}=h_{\text {limit_high }}-h_{\text {plane_adjust }}$

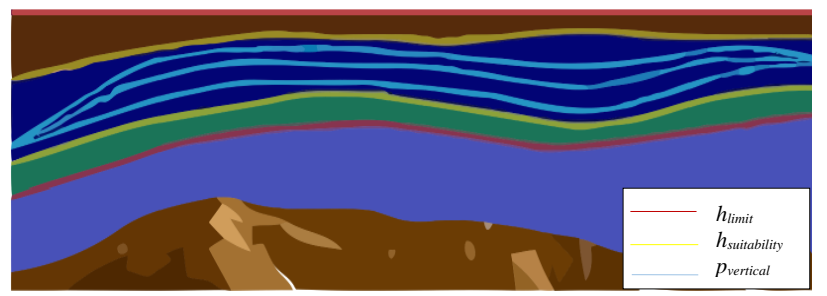

Fig. 2. Elevation dimension diagram of flight route planning

3) Population Characteristics

The population includes a certain number $\left(N_{p}\right)$ of solutions (i.e., antibodies). Each antibody represents a candidate flight path composed by a series of flight waypoints (i.e., genes). In this paper, we assume each candidate flight path including 10 flight waypoints, that is, $\left(N_{f}=10\right)$ connected together to form a path from one point to another (starting point $\rightarrow$ intermediate waypoints $\rightarrow$ ending point) shown in formula (6). The starting point (i.e. $P_{s}$ ) and ending point (i.e. $P_{e}$ ) are initialized with fixed values.

$$
\begin{aligned}
& P S=\left[\begin{array}{c}
P_{f}(1) \\
P_{f}(2) \\
\vdots \\
P_{f}(l) \\
\vdots \\
P_{f}\left(N_{p}\right)
\end{array}\right]=\left[\begin{array}{cccccc}
P_{f}(1,1) & P_{f}(1,2) & \ldots & P_{f}(1, k) & \ldots & P_{f}\left(1, N_{f}\right) \\
P_{f}(2,1) & P_{f}(2,2) & \ldots & P_{f}(2, k) & \ldots & P_{f}\left(2, N_{f}\right) \\
\vdots & \vdots & \ldots & \vdots & \ldots & \vdots \\
P_{f}(l, 1) & P_{f}(l, 2) & \ldots & P_{f}(l, k) & \ldots & P_{f}\left(l, N_{f}\right) \\
\vdots & \vdots & \ldots & \vdots & \ldots & \vdots \\
P_{f}\left(N_{p}, 1\right) & P_{f}\left(N_{p}, 2\right) & \ldots & P_{f}\left(N_{p}, k\right) & \ldots & P_{f}\left(N_{p}, N_{f}\right)
\end{array}\right] \\
& k=1,2, \ldots, N_{f}, N_{f}=10
\end{aligned}
$$

4) Selection technique

A roulette-wheel method is utilized in selection operation, which randomly and repeatedly selects a half of antibodies from its parent antibodies.

The main idea of roulette-wheel can be imagined a rotating wheel can only rotates turn around at most. Before each wheel disc put the chromophore randomly somewhere on the outer edge of the wheel disc, that is, the chromophore does not rotate with the wheel disc, and a random number $p_{c}$ represents its position. After the roulette rotates, the sector number of the roulette indicated $(j)$ by the dice changes constantly. When the roulette stops, the sector number on the roulette indicated by the dice $\left(p_{s}\right)$ is 
the individual number selected for this roulette, and the initial rotation position of disc expressed as $p_{\text {sum }}$.

The roulette-wheel method's selection process is as follows:

Tab. 1. Roulette-wheel method pseudo code

\begin{tabular}{ll}
\hline $\begin{array}{l}\text { Roulette-wheel method } \\
\text { pseudo code }\end{array}$ & Function \\
\hline for $i \leftarrow 1, i \in\{1,2, \ldots, n\}$ do & $\begin{array}{l}\text { The first to } i^{\text {th }} \text { dice throw } \\
p_{c} \text { is initialized as a random number } \\
\text { between } 0 \text { and } 1\end{array}$ \\
$p_{c} \leftarrow \operatorname{rand}(0,1)$ & $j$ is set as 1 \\
& The termination condition is satisfied \\
$p_{\text {sum }} \leftarrow 0$ & The wheel turns to new position $\left(p_{\text {sum }}\right.$ \\
$j \leftarrow 1$ & $+p(j))$ \\
while $p_{\text {sum }}<p_{c}$ do & $j$ is updated accordingly \\
$p_{\text {sum }} \leftarrow p_{\text {sum }}+p(j)$ & The wheel stops turning \\
$j \leftarrow j+1$ & $p_{s}$ goes back to $j-1$ \\
end while & End of the cycle \\
$p_{s}(i) \leftarrow j-1 ;$ & \\
end for & \\
\hline
\end{tabular}

5) Clone and mutation operator

A certain number of antibodies are cloned to prepare for the next generation's mutation operation. In this paper, all antibodies cloned ( i.e. '1-1 selection probability' ) are mutated to produce new generation child antibodies (path). Mutating antibodies are chosen randomly, which will improve the diversity of antibodies. The operations of selection, cloning and mutation operation is repeated until the stopping criteria are met.

\section{6) Vaccination Operation}

A vaccine is a short segment containing the most outstanding antibody, which is used to inoculate other antibodies. One antibody can be chosen as a vaccine when the antibody's probability exceeds preset threshold. In this paper, a vaccine is inoculated in three ways: Part of antibodies are randomly chosen to be inoculated, all antibodies to be inoculated and Each antibody to be inoculated but the inoculated position is random selected.

7) Population update technique

The affinity function can be measured by the antibody's comprehensive voyage, which is equivalent to the path's weighted cost. The different types of threats are transformed into terrain threats by their maximum influence range and height, whose affinity function can comprehensively express as follows:

$$
F(k)=\sum_{k=1}^{9} C d_{\text {seg }}(k)
$$

$C d_{s e g}(k)=\sqrt{\left(x_{f}(k+1)-x_{f}(k)\right)^{2}+\left(y_{f}(k+1)-y_{f}(k)\right)^{2}+\alpha \times\left(h_{f}(k+1)-h_{f}(k)\right)^{2} \times 10^{-6}}$ $k=1,2, \ldots, 9$

$$
\alpha=\left\{\begin{array}{cl}
10^{b}, b>0, b=\text { Constant } & \text { Threat }_{L}>\text { Threat }_{H} \\
10^{\circ} & \text { Threat }_{L} \approx \text { Threat }_{H} \\
10^{b}, b<0, b=\text { Cons } \tan t & \text { Threat }_{L}<\text { Threat }_{H}
\end{array}\right.
$$

Here, $F(i)$ is an affinity function of ith flight segment's comprehensive distance $C d_{\text {seg }}(i)$. In formula (8), $(x(i), y(i))$ and $(x(i+1), y(i+1))$ are the coordination of flight point $p(i), p(i+1)$ respectively. $\alpha$ and $b$ is the coefficient of flight path's height, a plus constant. $b$ 's value is depended on circumstance's complicate degree of mission. Threat ${ }_{L}$ and Threat $_{H}$ repress the threat projection on horizontal and height.

After vaccine's segment is initialized by randomly selected from vaccine but starting and ending points. Three different vaccination modes are adopted in this paper: (1) part of antibodies randomly are selected, (2) the $i^{\text {th }}$ and (antibodySum+1-i) ${ }^{\text {th }}$ antibodies are selected and (3) the $i^{\text {th }}$ and $(i+\text { antibodySum } / 2)^{t h}$ antibodies are chosed to be vaccinated, antibodySum is the total number of antibodies. Children antibodies is generated by inoculation copy of parent antibodies with vaccine.

8) Evaluation technique and convergence criteria

In this paper, the convergence criterions include maximum iterative generation and the deadline of planning time. That is, if either criterions is reached, program will cease.

9) Path planning major steps by AIA

The details of the AIA progress are:

Parameter initialization. Maximum and minimum turning angles, maximum and minimum turning gradient, flight speed, maximum voyage and the size of antibody (path) population, the probability of crossover $P_{e}$ and variation mode $M_{v}$ are initialized.

Flight path initialization. In this paper, the plane coordination of track point are initialized randomly value in the range of airspace by formula (10). Here, $\left(x_{t}, y_{t}\right)$ is temp coordination of path point. The function of round() and random()are used for expression of rounding and generating random numbers respectively.

$$
\begin{aligned}
& x_{t}=\operatorname{round}\left(\operatorname{random}(0,1)^{*}\left|x_{f s}-x_{f e}\right|\right), \\
& y_{t}=\operatorname{round}\left(\operatorname{random}(0,1)^{*}\left|y_{f s}-y_{f e}\right|\right) \\
& x_{f}=\left\{\begin{array}{cc}
\min \left(x_{s p}\right) & x_{t}<\min \left(x_{s p}\right) \\
\operatorname{random}(0,1)^{*}\left|x_{f s}-x_{f e}\right| & \max \left(x_{s p}\right)>x_{t}>\min \left(x_{s p}\right) \\
\max \left(x_{s p}\right) & \max \left(x_{s p}\right)<x_{t}
\end{array}\right. \\
& y_{f}=\left\{\begin{array}{cc}
\min \left(y_{s p}\right) & y_{t}<\min \left(y_{s p}\right) \\
\operatorname{random}(0,1)^{*}\left|y_{f s}-y_{f e}\right| & \max \left(y_{s p}\right)>y_{t}>\min \left(y_{s p}\right) \\
\max \left(y_{s p}\right) & \max \left(y_{s p}\right)<y_{t}
\end{array}\right.
\end{aligned}
$$

Affinity function. Affinity function express the value of path planned, whose size can measure safety and voyage of flight.

Terminal condition. The program's terminal condition chosen includes flight path's value and iterations. The main consideration factor is former for sake of safety.

Selecting operation. The roulette-wheel method is adopted for selecting operation. Different affinity probabilities are used to avoid falling into local optimum.

Crossover and mutation operation. Conventional crossover and mutation operations is performed according to the establishment crossover probability $P_{e}$ and the variation mode $M_{v}$ after the immune body is selected.

Although a minimal threat trajectory is generatered for an 
aircraft by AIA, which is a 3D Zigzag lineonnected by a set of segmented polyline having poor operability due to its flyability constraints. So smoothing operation should be executed on the path. Smoothing operation performed on three-dimensional track includes two step: fly-ability adjust operation and path smooth operation. Fly-ability adjust operation means aircraft's climb angle and turn angle. When aircraft's flight checked its climb angle and turn angle beyond its limit value, it must adjust the path due to safety. To summarized, turn angle and pitch angle constraints is used to adjust the planned path on level and vertical aspect respectively. Overall, the following research works have been done in process:

In the course of online flight, when flight path's angle surpasses the maximum turn angle, we should adjust the turn angle by formula (11). The coordinates of flight point associated is adjusted accordingly to adapt to this adjustment. The path should also be within the specific scope as the pitch angle, as shown in formula (12). Length of the shortest track segment is set no more than $5 \mathrm{~km}$ considering maneuver time of aircraft, as shown in formula (13):

$$
\begin{gathered}
\alpha=\left\{\begin{array}{cc}
60^{\circ} & \alpha \geq 60^{\circ} \\
\alpha & 60^{\circ}>\alpha>-60^{\circ} \\
-60^{\circ} & \alpha \leq-60^{\circ}
\end{array}\right. \\
\beta=\left\{\begin{array}{cc}
30^{\circ} & \beta \geq 30^{\circ} \\
\beta & -30^{\circ} \leq \beta<30^{\circ} \\
-30^{\circ} & \beta<-30^{\circ}
\end{array}\right. \\
\min \left(C_{\text {seg }}(k)\right)>=5 k m, k=1,2, \ldots, 9
\end{gathered}
$$

After this fly-ability adjust, Cubic spline curve is adopted to smooth above 3D flight path.

\section{EXPERIMENTS AND RESULTS}

In this research, AIA is used to plan path under a simulated environment with mountain threats compared with Genetic Algorithm (GA), which is recently becoming a more popular optimization method in the aircraft path planning community. Starting point and ending point was fixed at $(1 \mathrm{~km}, 1 \mathrm{~km}),(60$ $\mathrm{km}, 60 \mathrm{~km}$ ) for one aircraft path planning respectively. For two aircrafts path planning, two initial coordination are set at $(1 \mathrm{~km}$, $8 \mathrm{~km})$ and $(10 \mathrm{~km}, 1 \mathrm{~km})$; while the destination coordination are still preset at $(60 \mathrm{~km}, 60 \mathrm{~km})$. In this paper, experiments were run on a Pentium (R) 2 with a CPU speed of $2.2 \mathrm{GHz}$. Other flight parameters are: Pitching angle $\in\left(-30^{\circ},+30^{\circ}\right)$; Maximum turning angle $\in\left(-60^{\circ},+60^{\circ}\right)$; Minimum flight segment length not greater than $5 \mathrm{~km}$; Velocity range $\in(61 \mathrm{~m} / \mathrm{s}, 77 \mathrm{~m} / \mathrm{s})$; airspace assumed $60 \mathrm{~km} \times 60 \mathrm{~km}$.

Three simulated scenarios were designed within an airspace to verify the AIA method's robustness and efficiency. In these tests, aircraft's number and threats' number (and/or types) are also changed. The following three tests provides a comparison of AIA and GA on planning path.

\section{A. Simulation Scenario \#1}

Figure 3(a) and 3(b) shows the plane path, 3D flight path planned by AIA. The path generated can satisfactorily avoid threats, whose plane path is the project of 3D flight path on horizontal. The threat zones are denoted as a set of colored contour graphs, which represent the elevation of the mountains. The aircraft fly from starting point on the bottom left corner to ending point on the top right corner. The planned path stays away from the threat with lower value. Figure 4 provides the simulation results planned with GA under the same environment. The results show that the GA is also capable of designing a feasible flight path in a shorter time (GA 3.875 seconds and AIA 4 seconds), but with a longer flight length than the AIA. This means that GA can quickly design a flight path under terrain threat conditions. This advantage helps the operator to decide how to address emergency cases with terrain threats. The operator can evaluate the two methods' merits and demerits and select an appropriate one for a particular mission.

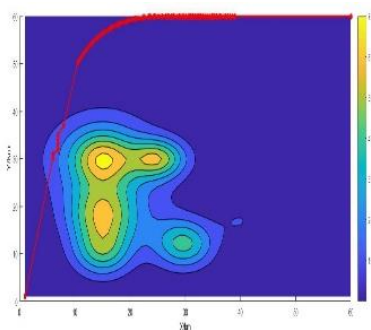

(a)

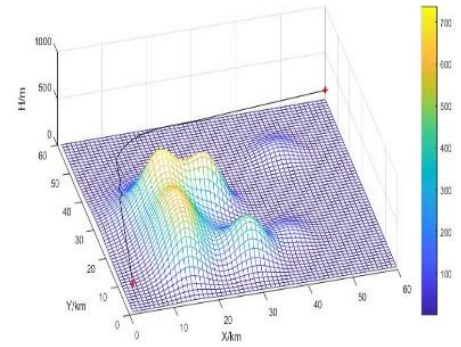

(b)
Fig. 3. Path on $x-y$ plane (a) and in 3D airspace (b) by AIA in simple circumstance

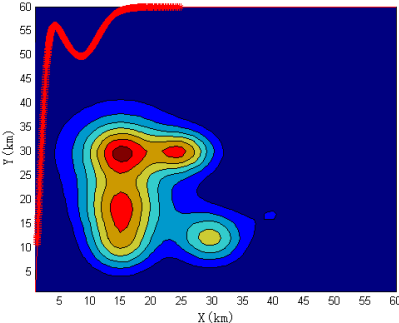

(a)

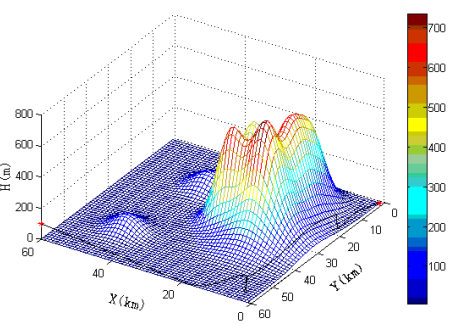

(b)
Fig. 4. Path on $x-y$ plane (a) and in 3D airspace (b) planned by GA under simple circumstance

\section{B. Simulation Scenario \#2}

The second test scenario examined four threats situated in the way of the aircraft reference path shown in Figure 5. Additionally, five new threats are set as no-fly-zone. These threats and their threat zone are represented as several colored cylinders in 3D airspace, and white rings on $x-y$ plane respectively.

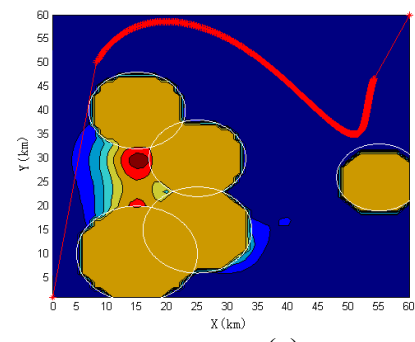

(a)

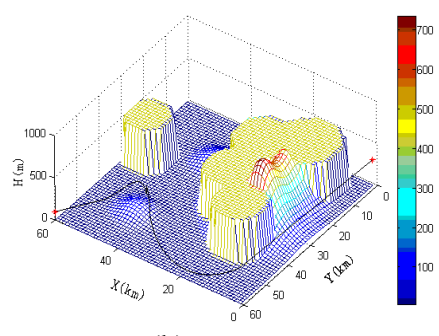

(b) 
Fig. 5. Path on $x-y$ plane (a) and in 3D airspace (b) by AIA in complicate circumstance

It can be seen that the path planed has bypassed these no-fly-zone and gone through low-lying areas from Figure 5. This shows that Artificial Immune Algorithm is feasible and effective in solving the problem of disaster rescue in complex environment.

The 2D and 3D paths are shown by AIA in Figure 5, which was successful in satisfying its objective requirements. The red path prior to a top priority on safety than minimize additional fuel usage and maximize reconnaissance.

Figure 6 shows the path planned by GA without avoiding the no-fly-zone due to its inefficacy at maintaining antibody diversity. By compared Figure 5 and Figure 6, AIA has higher success rate than GA under a complex environment.

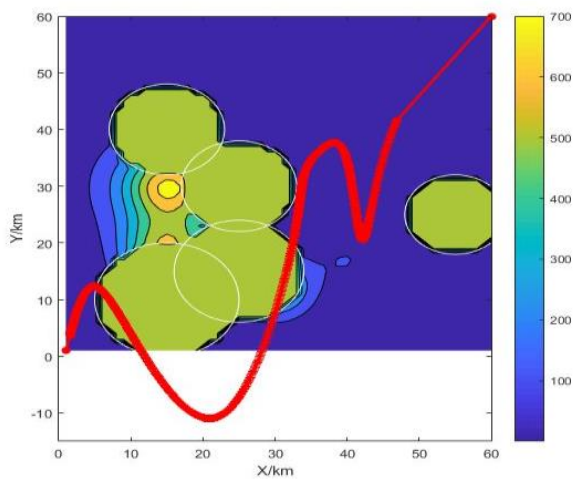

(a)

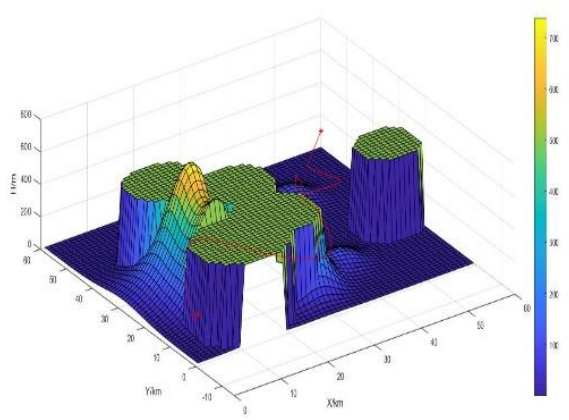

(b)

Fig. 6. Path on $x-y$ plane (a) and in 3D airspace(b) by GA in complicate circumstance

\section{Simulation Scenario \#3}

This scenario add more terrain and a pop-up threat near destination in simulation Scenario \#2. The result of planning flight path is shown on Figure 7 and Figure 8. The conclusions are like those from simulation scenario \#2: the path(s) is (or are) planned with the minimum flight value but also having the maximum safety margin against these ground obstacles. Figure 7 and Figure 8 also indicate AIA has a faster and more successful path planning ability than GA under a complicated environment to make a long story short.

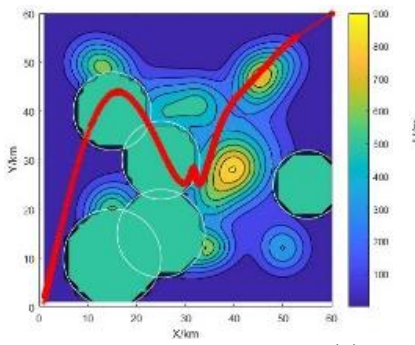

(a)

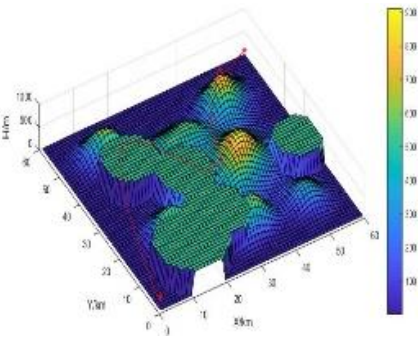

(b)

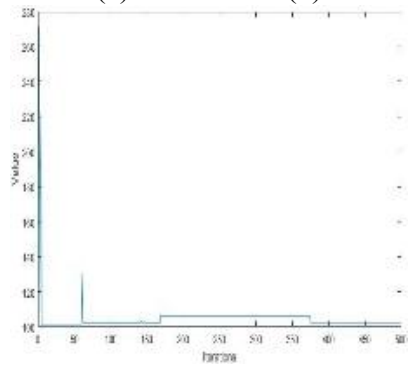

(c)

Fig. 7. Path on $x-y$ plane (a) and in 3D airspace (b) for two aircrafts by GA in complicate circumstance

In this research, the total aircraft flight path's length is planned as shortly as possible on the premise of safe flight. As a result, two airplane can easily reach the same estimation by slightly changing their velocities. In experiment, four threats are added into above environment, which expressed by cylinders Figure 7(b), 8(b) and shown in Figure 7(a) 8(a) by white circular zones.

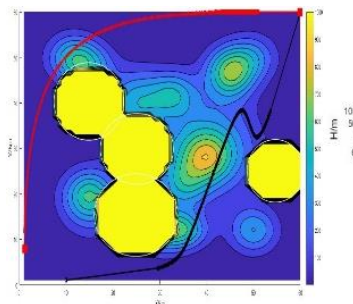

(a)

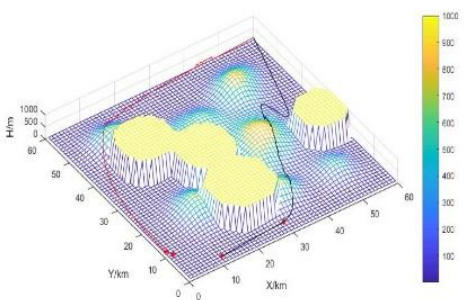

(b)

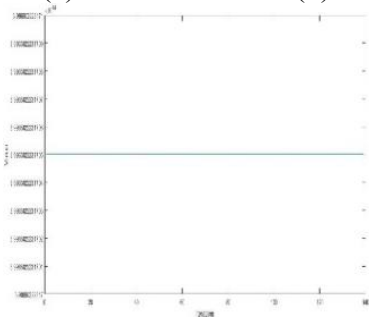

(c)

Fig. 8. Path on $x-y$ plane (a) and in 3D airspace (b) and replaced sub-optimal paths (c) for two aircrafts by AIA in complicate circumstance with no-fly-zone

In the iterative process of genetic algorithm, the instability of cost change shows that there is great randomness in the search of genetic algorithm in complex environment leading to converge difficultly to the optimal solution in figure 7(c). From Figure 7(a), 7(b), we can see that the newly designed path completely avoids these threats by GA but not bypass no-fly zone. As a result, GA failed in this experiment.

By using the AIA, two airplane's paths are designed passing safe area, which improves the successful ratio for aircraft rescue in Figure 8. There is $3.73 \mathrm{~km}$ 's distance difference 
between two aircrafts' flight paths in this simulation. The individual flight path length for two aircrafts in red color are $92.168 \mathrm{~km}$ for aircraft 1 and $88.438 \mathrm{~km}$ for aircraft 2 in black color.

\section{The analysis of iteration and antibody number's} influence

Based on Scenario \#1, experiments is carry out with the variation of iteration number and antibody number as constant. Each experiment is performed five times for every iteration number. The average value is taken as the last result, and the iteration number is increased from 10 to 150 with step of 20 . Figure 9(a) shows that the comprehensive cost is proportional to the iteration number. Moreover, from the comprehensive cost fitting line, the total cost does not increase with iteration numbers increased. Figure 9(b) shows that the planning time also becomes longer as the iteration number increases from $107.5 \mathrm{~s}$ to $109.4 \mathrm{~s}$. Therefore, it can conclude that the flight path difference between the two aircraft clearly decreases with the iteration number increased in figure 9(c).

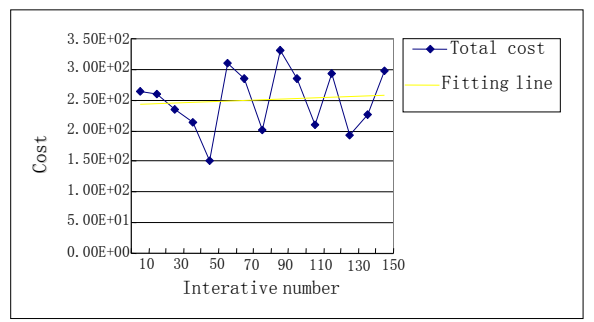

(a)

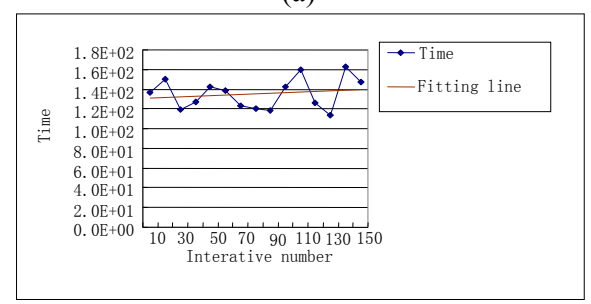

(b)

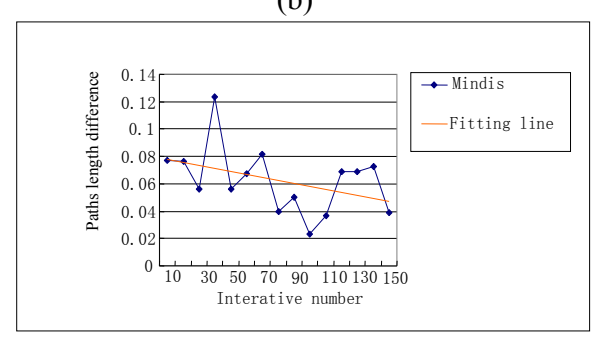

(c)

Fig. 9. (a) Cost table; (b) Time table; (c) Two path difference table with iterative number increased

To completely depict the influence of the iteration number, we provide a function (TC, Total Cost for abbreviation) containing the above three relative parameters: planning time (time), comprehensive cost (cost) and two flight path difference $(P d)$ :

$$
T C=\text { time }^{*} \cos ^{*} P d
$$

where time is expressed as the time value in figure 9 (a), cost is the comprehensive threats' influence shown in figure 9(b) and $P d$ is the difference between the two planned paths, shown in figure 9(c). The result $T C$ with iterative number increased is shown in Figure 10. It can be seen $T C$ has gone through three stages of change with iteration increased: slight increasing process with iteration number less than 10 , largely declining process with iteration number in range of $(10,80)$, and slow improvement process when iteration number larger than 80 . This phenomenon indicates that there is a suitable iteration algebra to minimize the flight cost.

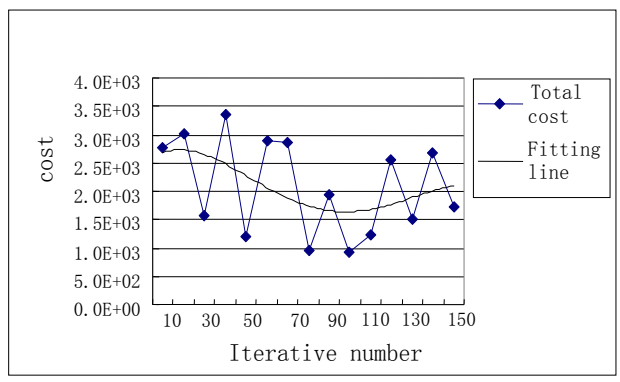

Fig. 10. Total cost with iterative number increased

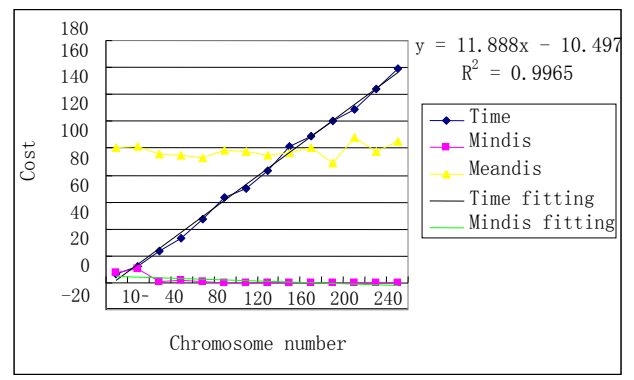

(a)

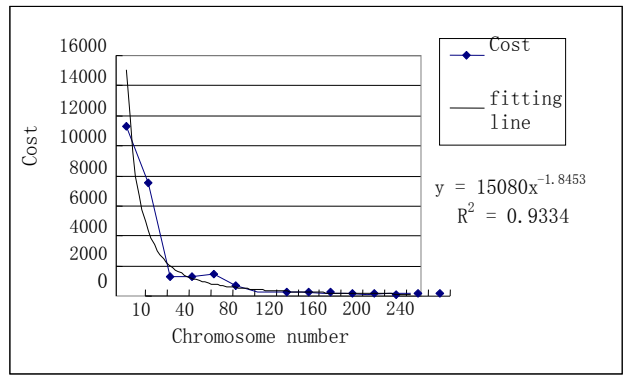

(b)

Fig. 11. (a) Parameters influence, (b) Cost with antibodies number increased

Figure 11(a) shows the effect of the three influence parameters including planning time (time) and comprehensive cost (cost) as the antibody numbers are increased. The flight path difference $(P d)$ of two aircraft decreases with antibody less than 90 , but changes slightly when antibody larger than 90 . Time increases in ratio to antibody number, which means an increase of antibodies' number will greatly make the path planning time greatly extended. Figure 11(b) shows that comprehensive cost exhibits a larger decrease with antibody number increased, whose fitting line approximately follows an exponent function. This shows the remarkable influence that improving the antibody number has on the total cost value. When antibody number is larger than 120, the downtrend of cost's fitting line become slow. 


\section{CONCLUSIONS AND DISCUSSIONS}

This paper studies the path planning of single and double aircraft in complex rescue environment. AIA-based method is applied in solving the problem of path planning in complex environment. With adding aircraft's fly-ability and operation model, AIA method can successfully design flight path under complicate environment even congested with no-fly-zone or moving theat. In this paper, three simulation scenarios is adopt to study AIA's path planning' feasibility with comparison with genetic algorithm (GA) commonly used in aircraft path planning field. Through the comparison of the first two experiments, AIA has better performs than GA under complex circumstances.

In order to study the influence of iterative number and the number of antibodies on the performance of path planning of artificial immune algorithm, we introduce path planning's total cost as an index to measure the advantages and disadvantages of path.

The experiments shows total cost changed with approximate parabola law as the iteration number increased and there is least total cost at the number of 90 . Total cost also decreased with antibody number increased and there is little change when antibody number larger than 120 .

Although artificial immune algorithm can complete route planning in complex environment, the influence of climate factors such as wind mutation on flight maneuverability and adaptability of route planning methods still needs further study. The problems of route planning of multi aircraft formation and dynamic planning of how to cooperate with various types of aircraft to maximize rescue efficiency need to be improved in the next research work to improve and perfect.

\section{ACKNOWLEDGMENT}

This work was supported in part by ZR2019BD036 (Doctor's fund of Shandong Natural Science Foundation).

\section{REFERENCES}

[1] B. Ibrahim, H. Noura, Formation Flight Control of Multi-UAV System using Neighbor-based Trajectory Generation Topology, WSEAS Transactions on Applied and Theoretical Mechanics, ISSN / E-ISSN: 1991-8747 / 2224-3429, Volume 15, 2020, Art. \#20, pp. 173-181.

[2] Hien N. V., Diem P. G., A Model-based Design to Implement Controllers for Quadrotor Unmanned Aerial Vehicles, WSEAS Transactions on Systems, ISSN / E-ISSN: 1109-2777 / 2224-2678, Volume 18, 2019, Art. \#6, pp. 45-61.

[3] N. Kayhani et al. "Heavy Mobile Crane Lift Path Planning in Congested Modular Industrial Plants using a Robotics Approach", Automation in Construction, vol. 122, pp. 1-17, 2021.

[4] X. B. Yu, C. L. Li, and G. G. Yen. "A Knee-Guided Differential Evolution Algorithm for Unmanned Aerial Vehicle Path Planning in Disaster Management", Applied Soft Computing, vol. 98, pp. 1-15, 2021.

[5] X. Zhong, J. Tian, H. Hu, et al. "Hybrid Path Planning Based on Safe A* Algorithm and Adaptive Window Approach for Mobile Robot in Large-Scale Dynamic Environment", Journal of Intelligent \& Robotic Systems, vol. 99, pp. 65-77, 2020.

[6] Sang H., You Y, Sun X, et al. The Hybrid Path Planning Algorithm based on Improved $\mathrm{A}^{*}$ and Artificial Potential Field for Unmanned Surface Vehicle Formations [J]. Ocean Engineering, 2021(223):1-16.
[7] L. Larsen, J. Kim, "Path Planning of Cooperating Industrial Robots using Evolutionary Algorithms", Robotics and Computer-Integrated Manufacturing, vol. 67, no.1, pp. 1-16, 2021.

[8] Y. Ma, Z. Y. Mao, T. Wang, et al., "Obstacle Avoidance Path Planning of Unmanned Submarine Vehicle in Ocean Current Environment based on Improved Firework-Ant Colony Algorithm", Computers \& Electrical Engineering, vol. 87, no.1, pp. 1-14, 2020.

[9] Z. Liu, X. Yuan, G. Huang et al., "Two Potential Fields Fused Adaptive Path Planning System for Autonomous Vehicle under Different Velocities", ISA Transactions, (in press), 2020.

[10] I. Sung, B.J. Choi, P. Nielsen, "On the Training of a Neural Network for Online Path Planning with Offline Path Planning Algorithms", International Journal of Information Management, vol. 57, pp. 1-9, 2021.

[11] Zhou Y, Su Y, Xie A et al., "A Newly Bio-inspired Path Planning Algorithm for Autonomous Obstacle Avoidance of UAV", Chinese Journal of Aeronautics, vol. 1, pp. 1-11, 2021.

[12] J. K. Wang, B. P. Li, M. Q. H. Meng, "Kinematic Constrained Bi-directional RRT with Efficient Branch Pruning for Robot Path Planning", Expert Systems with Applications, vol. 170, pp. 1-7, 2021.

[13] G. Pradeep Kumar, B. Sridevi, Simulation of Efficient Cooperative UAVs using Modified PSO Algorithm, WSEAS Transactions on Information Science and Applications, ISSN / E-ISSN: 1790-0832 / 2224-3402, Volume 16, 2019, Art. \#11, pp. 94-99.

[14] Z. Wang, G. Li and J. Ren, "Dynamic Path Planning for Unmanned Surface Vehicle in Complex Offshore Areas Based on Hybrid Algorithm" , Computer Communications, vol. 166, pp. 49-56, 2021.

[15] R. Sarkar, D. Barman and N. Chowdhury, "Domain Knowledge Based Genetic Algorithms for Mobile Robot Path Planning Having Single and Multiple Targets", Journal of King Saud University - Computer and Information Sciences, (in press).

[16] C. Lamini, Said Benhlima, A. Elbekri, "Genetic Algorithm Based Approach for Autonomous Mobile Robot Path Planning", Procedia Computer Science, Vol. 127, pp. 180-189, 2018.

\section{Creative Commons Attribution License 4.0 (Attribution 4.0 International, CC BY 4.0)}

This article is published under the terms of the Creative Commons Attribution License 4.0 https://creativecommons.org/licenses/by/4.0/deed.en US 